\title{
Sexualidad al debate. Transformaciones, tensiones y continuidades en la historia reciente de Costa Rica
}

\author{
Sexuality to debate. Transformations, tensions and \\ continuities in recent Costa Rican history
}

\author{
Doris Fernández Carvajala \\ doris.fernandez.carvajal@una.cr \\ María Luisa Preinfalk Fernández ${ }^{\mathrm{b}}$ \\ maria.preinfalk.fernández@una.ac.cr \\ Paula Sequeira Rovira ${ }^{\mathrm{C}}$ \\ paula.sequeira.rovira@una.cr \\ a,b,c Instituto de Estudios de la Mujer, Universidad Nacional, Costa Rica
}

Recibido: 17/02/2020 • Aceptado: 30/10/2020

\begin{abstract}
Resumen
El presente artículo refiere algunas discusiones que se han producido en Costa Rica, en los últimos 50 años, en torno a temáticas relacionadas con la sexualidad. Se analizaron acontecimientos que marcaron el rumbo de la historia en este campo, sus repercusiones y los retos que generaron. Varias de estas disputas giran en torno a la educación en sexualidad, las enfermedades de transmisión sexual, la planificación familiar, la sobrepoblación, el aborto y otros temas atinentes. En estas discusiones, se destacaron diferentes actores sociales, con múltiples enfoques o visiones que, muchas veces, se contrapusieron, cuyas intervenciones suscitaron tensiones en el ambiente político nacional y, en algunas ocasiones, debilitaron las iniciativas estatales. La revisión y análisis de la información se efectuó a partir de periódicos nacionales, investigaciones,
\end{abstract}


informes de proyectos, libros y artículos académicos que se publicaron entre 1965 y 2019. Los análisis realizados permiten afirmar que las discusiones en sexualidad y reproducción han tenido un lugar protagónico en la visión de desarrollo del país, de allí la importancia de contextualizar las dinámicas sociales vinculadas a ellas y las implicaciones que tienen en la vida de las personas.

Palabras clave: Sexualidad; Costa Rica; historia; educación sexual; aborto

\section{Abstract}

This article refers to some discussions that have taken place in Costa Rica in the last 50 years, regarding issues related to sexuality. Events that marked the course of history in this field, its repercussions and the challenges they generated were analyzed. Several of these disputes revolve around sexual education, sexually transmitted diseases, family planning, overpopulation, abortion and other relevant issues. In these discussions, different social actors were highlighted, with multiple approaches or visions that were often opposed and whose interventions raised tensions in the national political environment and sometimes weakened state initiatives. The review and analysis of the information was carried out based on national newspapers, scientific research, project reports, books and academic articles, which were produced between 1965 and 2019. The analyzes carried out lead to affirm that the discussions in sexuality and reproduction have had a leading place in the country's vision of development, hence the importance of contextualizing the social dynamics linked to them and the implications they have in people's lives.

Key words: Sexuality; Costa Rica; history; sexual education; abortion

\section{INTRODUCCIÓN}

La sexualidad se presenta como un tema que convoca intensas reflexiones tanto a nivel nacional como internacional. Las pasiones que genera hacen que su debate se cuele en todo tipo de espacios como cenas familiares, seminarios académicos, conferencias de prensa, posgrados universitarios, 
discursos presidenciales, entre muchos otros. Sus posibilidades analíticas son interminables y los diversos enfoques que la tratan conviven, en algunos casos, de forma armoniosa, aunque también pueden generar enfrentamientos profundos, tal y como ha ocurrido, por ejemplo, dentro del propio movimiento feminista (Allen, 2001). A pesar de que constantemente se producen discusiones sobre la sexualidad, regularmente se la suele considerar desde perspectivas esencialistas, sin importar el contexto o la historia. En otras ocasiones, se dan por sentado experiencias, en las que no se profundiza. En todo caso, desconocer el pasado o presente incide en cometer apreciaciones ligeras sobre generaciones anteriores y la propia vida.

Llegar a entender que el pasado sexual y reproductivo de las personas costarricenses está influenciado por múltiples presiones nacionales e internacionales puede ser muy provechoso, pues en las reflexiones sobre estos temas es importante contextualizar su discusión en un marco más o menos delimitado de análisis. Esta contextualización permite interpretar el pasado, entender mejor el presente y prepararse para situaciones del futuro. Con esto en mente, habría que decir que, al hablar de la sexualidad de hoy en día, los análisis que se hagan deben tomar en cuenta importantes acontecimientos que se produjeron desde mediados de la década de 1960. A partir de ahí, Costa Rica ve aparecer toda una constante institucionalización y preocupación estatal por la sexualidad y la reproducción, tal y como se probará más adelante. Lo anterior produjo que sobre estas categorías recayera una asidua mirada desde la prensa, la política internacional, las decisiones de los gobiernos y, probablemente, también las discusiones más íntimas que se producían entre las personas.

El objetivo de este artículo es presentar algunas acciones desarrolladas, desde los gobiernos de turno y organismos no gubernamentales, que marcaron la construcción del camino que tomó el país en temáticas sobre la sexualidad y la reproducción, así como entender sus repercusiones y retos actuales. En este sentido, se pretende esbozar un breve análisis sobre los ejes en torno a los cuales han girado parte de las discusiones en el país, en relación con la sexualidad, en los últimos cincuenta años. Varias de estas disputas han abordado temas como la educación en sexualidad, las enfermedades de transmisión sexual, la planificación familiar o la sobrepoblación, solo para mencionar algunas de las preocupaciones más llamativas. Los debates de todas estas situaciones han generado posiciones 
disímiles, con participación de diferentes actores sociales, quienes han expuesto sus argumentos a favor y en contra y cuya discusión se mantiene vigente hoy en día.

A continuación, se aborda parte de estos debates, a partir de la revisión y análisis de algunos periódicos nacionales, tesis, informes de proyectos, libros y artículos académicos que se publicaron entre 1965 y 2019. Específicamente sobre la revisión de periódicos, se examinó La República para el apartado del siglo XX a través del Sistema Nacional de Bibliotecas (SINABI) pues es el único diario que mantiene una constancia durante este periodo. En el caso de los periódicos revisados durante el siglo XXI (Semanario Universidad, La República y La Nación), se recopilaron a través de las bases de datos de las páginas en Internet de cada uno. A partir de esta información, se realizó una selección de algunos de los múltiples debates presentados durante dichas épocas para fortalecer la generación de deliberaciones sobre estos asuntos. Las discusiones que aquí se reúnen son un resumen de parte de los hallazgos del proyecto de investigación Debates sobre salud sexual y la salud reproductiva en Costa Rica que se realiza desde el Instituto de Estudios de la Mujer de la Universidad Nacional.

\section{ENTRE LA REVOLUCIÓN SEXUAL Y EL CAMBIO DE SIGLO}

La sexualidad como categoría de análisis ha sido abordada desde muy diferentes campos. Una de las posiciones más ampliamente citadas se encuentra en los postulados trabajados en Historia de la Sexualidad I, por Michel Foucault (2009). A partir de allí, se ofrecen pistas para su estudio y comprensión. Buscando alejarse de las hipótesis que planteaban que sobre la sexualidad no se habla, este teórico francés propuso que, desde el siglo XIX, las sociedades occidentales vieron aparecer una amplia variedad de discursos sexuales en campos tan variados como la medicina, la religión, la arquitectura o el psicoanálisis. Como parte de esta misma teoría, se estableció que para entender al ser humano como sujeto sexual, un elemento clave que también ha producido discursos es la confesión, la cual se convirtió en una técnica que se ha interiorizado para obtener el conocimiento de una supuesta verdad de la que se es parte (Foucault, 2009; Foucault, 2001).

92

Doris Fernández Carvajal, María Luisa Preinfalk Fernández, Paula Sequeira Rovira

\section{()(1)(2)}


Parte del proceso que le otorgó una importancia suprema a la sexualidad, fue apoyado por los sexólogos del siglo XIX y XX, quienes jugaron un papel fundamental en el desarrollo de conceptos y normalidades en esta parte de la vida humana. Pasando por Krafft-Ebing, Kinsey o Masters y Johnson, la sexualidad fue tomando un carácter fuertemente científico (Foucault, 2009) que buscó hacer mediciones o crear estándares de procesos tan variados como, por ejemplo, el orgasmo. Estas investigaciones, así como los comprimidos contraceptivos que se distribuyeron a partir de los años sesenta (Tone, 2002), facilitaron un cuestionamiento a las vivencias eróticas y apoyaron ideas relacionadas con la llamada revolución sexual.

En este caso, la revisión de ciertos acontecimientos que sucedieron a finales del siglo XX, se convierte en una necesidad para quienes se interesan por el análisis de la sexualidad en Costa Rica. Al menos desde mediados de los años sesenta en adelante, se produjo un claro interés por institucionalizar y vigilar las conductas reproductivas, así como argumentar con persistencia acerca de la sexualidad costarricense (Madrigal \& Sosa, 1987; Rosero, 1981a; Rosero, 1981b; Rodríguez de Ortega, 1978). Por aquellos años, se hablaba continuamente sobre la educación sexual, los métodos anticonceptivos modernos, las implicaciones de un país que no limitaba la descendencia, los problemas religiosos asociados con los anticonceptivos (previos y posteriores a la impresión de la Encíclica Humanae Vitae de 1968), el esparcimiento de las llamadas enfermedades sexuales o el aborto. Asimismo, algunos años después, a finales de la década de 1980 y en 1990, el país también se empezaría a preocupar por el embarazo en niñas y adolescentes, la pornografía, la esterilización anticonceptiva, la concepción en tubos de ensayo, el Síndrome de Inmunodeficiencia Adquirida (Elizondo et al., 1993; Sandoval \& López, 1990; Asociación Demográfica Costarricense, 1987).

Con mucha regularidad, las discusiones sobre la sexualidad y la reproducción pusieron a las mujeres en un lugar complejo, generando múltiples disputas y controversias con actores e instituciones que expresaban todo tipo de posturas sobre la capacidad reproductiva y la conveniencia de gestionar adecuadamente la descendencia. Algunos de los actores se mostraron recelosos de ciertas perspectivas de control de la reproducción; otros se manifestaron más preocupados por la educación o por la natalidad, pero, generalmente, los argumentos que esbozaban fueron parte de procesos más amplios que acontecían a nivel internacional (Tone, 2002). Por ejemplo, en 
las décadas finales del siglo XX, aparece una serie de eventos importantes que modificaron o influyeron en la forma en cómo hoy se conciben la sexualidad, los derechos reproductivos o la posibilidad de las consignas de autodeterminación de las vidas eróticas de las personas. Asimismo, la década de 1960 y 1970 trajo consigo un desarrollo de los postulados del movimiento feminista mundial y un establecimiento de los primeros grupos feministas en el país (Carcedo et al., 1997). Esto, sin duda, generó un espacio de referencia teórica particular sobre las vivencias sexuales de las personas, pero, sobre todo, de las mujeres y sus cuerpos.

El atractivo por institucionalizar la reproducción o la educación sexual en el país tendrá muy diversos intereses (preocupaciones poblacionales, feministas, religiosos, etc.). Estas organizaciones vendrán de todos los campos como ministerios, asociaciones religiosas, universidades y demás instancias de la sociedad civil. Los anteriores serán momentos en que tanto gobernantes, como sectores no gubernamentales, nacionales e internacionales, empezarán a poner una mirada de mayor detenimiento en la intimidad de las personas y sus implicaciones sociales, preocupados por presiones internacionales que alertaban sobre la sobrepoblación de los llamados países subdesarrollados (Connelly, 2008; Tone, 2002). Dos espacios fundamentalmente importantes desde el sector gubernamental que surgieron por aquellos años fueron el Programa Nacional de Planificación Familiar y Educación Sexual, en 1968 (Rosero, 1981a), el cual buscó influir en aspectos demográficos, educativos y relativos a la anticoncepción, y la Asesoría de Educación Sexual del Ministerio de Educación Pública (MEP), que inició labores en 1970 (Barth, 1988).

Otras instancias en el ámbito no gubernamental que surgieron fueron el Centro de Orientación Familiar (COF), fundado en 1968, y el Centro de Integración Familiar (CIF), que inició actividades en 1971, los cuales provenían de la Iglesia Anglicana y de la Iglesia Católica, respectivamente. Asimismo, la Asociación Demográfica Costarricense (ADC), creada en 1966, será muy importante en el fomento de capacitaciones, investigaciones y recopilación de datos sobre temáticas atinentes. Por otra parte, el Centro de Estudios Sociales y de Población (CESPO), adscrito a la Universidad de Costa Rica (UCR), inició en 1968, aportando a la investigación en el campo de temáticas poblacionales.

\section{4}

Doris Fernández Carvajal, María Luisa Preinfalk Fernández, Paula Sequeira Rovira

\section{()(1)(2)}


No se puede dejar de consignar que, detrás de esto, muchas veces había influencia o apoyo ideológico de instancias poderosas como la Fundación Ford, la Agencia para el Desarrollo Internacional (AID), el Centro Rockefeller, International Planned Parenthood Federation (IPPF), el Banco Mundial, el Consejo de Población (Population Council), el Programa de las Naciones Unidas para el Desarrollo (PNUD) o el Fondo de Población de las Naciones Unidas (UNFPA) (Connelly, 2008). Estos no solo invirtieron grandes sumas de dinero en países de América Latina, entre los que Costa Rica no fue la excepción, sino que también apoyaron investigaciones y promovieron asistencia técnica para desarrollar capacitaciones (Castro, 2015; Felitti, 2009). Dichos apoyos económicos permitieron el desarrollo y publicación de encuestas nacionales de reproducción y fecundidad tal y como se expresa en estos documentos (White et al., 1992; ADC, 1987; Rosero, 1981b; Dirección General de Estadística y Censos, 1978).

Una de las temáticas sobre las que más énfasis se puso fue el control de la natalidad. Los gobiernos, tanto nacionales como los de otros países, empezaron a mostrar un interés especial por buscar alternativas para evitar el punto en el que la sobrepoblación produjera que los recursos (ambientales, alimenticios u otros) no alcanzaran para satisfacer las necesidades presentes y futuras. Desde principios de los años sesenta, en el país comenzaron a desarrollarse estudios sobre prevalencia anticonceptiva y fecundidad (Madrigal \& Sosa, 1987), los cuales daban insumos para, entre otras acciones, reconocer científicamente el problema del crecimiento poblacional desordenado. Tanto en Costa Rica como a nivel internacional se hablaba de la bomba poblacional, idea que fue popularizada (mas no inventada) por Paul Ehrlich en 1968 con propósitos neo maltusianos. Ehrlich era un entomólogo estadounidense que pensaba que el incremento de la natalidad, no solo aquella que era no reflexionada, sino también que no estuviera determinada por cada gobierno alrededor del mundo, comprometía la vida en la Tierra y representaba daños irreparables para el futuro de la humanidad. Para Ehrlich, la solución para este caos humano y planetario se podía resumir en mantener un equilibrio de "la relación entre la tasa de natalidad y la tasa de mortalidad" (Ehrlich, 1969, p. 27) y lograr que los nacimientos no fueran mayores que las defunciones.

Los referentes conceptuales con tintes bélicos que estaban detrás de la bomba poblacional también apoyaban otra de las expresiones populares de aquellos años, como lo fue la explosión demográfica. Ambas suponían 
que, si no se hacía nada por remediar la sobrepoblación, las consecuencias serían fatídicas para los llamados países subdesarrollados, pero también para los desarrollados. Por estos motivos, para promover el progreso de un país, primero había que influir sobre su sexualidad y su reproducción. Este tipo de frases, y otras similares, eran comunes en Costa Rica, según el vocabulario y los análisis que se hacían desde un periódico como La República.

Para contrarrestar esta situación, tanto el Estado como otras instancias no gubernamentales buscaron establecer el concepto de la llamada planificación familiar responsable entre las parejas. Aunque, los orígenes de iniciativas privadas que intentaron introducir anticonceptivos en el país datan de la década de 1950 (González, 1985), sería hasta finales de 1960 cuando el Estado tomaría un rol más activo, con la creación del Programa Nacional de Planificación Familiar y Educación Sexual y con la importación de píldoras y dispositivos intrauterinos para ser distribuidos por la seguridad social. Otros métodos anticonceptivos, como la esterilización, tardarán más en hacerse parte de las opciones accesibles a la población. Si bien se sabe que este tipo de operaciones ya se realizaban en la década de 1940 (Carranza, 2004), las inquietudes que generaba un procedimiento tan definitivo no permitieron su democratización sino hasta 1999, cuando teóricamente comenzó a estar a disposición de cualquier persona mayor de edad. Sin embargo, algunas investigaciones mostraron que el personal de salud seguía generando barreras para cierto tipo de mujeres, a las que veían como no aptas para este procedimiento (Maroto et al., 2004; Fernández \& Sequeira, 2011).

Para contrarrestar el desorden poblacional (explosión demográfica) y para ofrecer herramientas de dominio sobre los momentos y precisiones necesarias para entablar intimidad corporal con otra persona (edad, estado civil, orientaciones permitidas, condiciones emocionales necesarias), se planteó la importancia de ofrecer educación sexual en escuelas y colegios. Antes de que el Estado costarricense comenzara a interesarse por estos temas formalmente en 1969 (González, 1991, p. 6A), dicha instrucción fue fomentada por diversas instancias no gubernamentales como la ADC, el COF o el CESPO, entre otros. Las acciones de educación no formal se llevaban a cabo por diversos sectores y carecían de una estrategia e impacto a nivel nacional.

96

Doris Fernández Carvajal, María Luisa Preinfalk Fernández, Paula Sequeira Rovira

\section{()(1)(2)}


Por ejemplo, de la variedad de cursos de esta índole que se ofrecieron en la década de 1970, uno de ellos fue el "primer curso centroamericano de capacitación básica en demografía y educación sexual", organizado por el Centro Latinoamericano de Demografía (CELADE), el CESPO y el Comité de Servicio de los Amigos. El curso se interesaba en capacitar a docentes centroamericanos durante cinco semanas (La República, 1970, p. 6); el Ministro de Educación de ese momento, Víctor Brenes Jiménez, inauguró el evento. Los temas tratados fueron: a) principios básicos de la educación sexual; b) anatomía genital; c) fisiología de la reproducción; d) antropología sexual; e) amor sexual e institución matrimonial, amor y fecundidad, paternidad responsable; f) las etapas evolutivas de la sexualidad; g) psicología del adolescente; h) amor y amistad; i) enfermedades venéreas; j) homosexualismo y otras patologías sexuales; k) paternidad responsable, planificación familiar (La República, 1970, p. 21). No está de más decir que, durante aquellos años, la homosexualidad era vista como un desorden del comportamiento cercano a conductas delictivas y peligrosas para la sociedad (Sequeira, 2020a), por lo que enseñar al "homosexualismo" como una "patología sexual" era parte de la lógica de cómo se concebía esta expresión de la sexualidad.

Los planes para extender a todo el territorio costarricense la educación sexual estuvieron marcados por la controversia, pues muchas veces, posturas divergentes buscaban poner el acento teórico o metodológico en diferentes temas. Uno de los actores más destacados, que generalmente intentaba ser un contrapeso a posiciones que le parecían moralmente inadecuadas fue la iglesia católica. Sin embargo, tal y como se puede comprobar en diversos pronunciamientos de figuras religiosas del ámbito costarricense de aquellos años, más que buscar la desaparición de la educación sexual, la influencia religiosa buscó dejar clara la necesidad de que este tipo de educación estuviera marcada por la ciencia y la moral, con el fin de evitar la incitación de ciertas conductas o acciones que consideraba desordenadas (Fernández, 1992, p. 5A; Valverde et al., 1991, p. 4A; La República, 1977, p. 13). Exaltar el valor de la ciencia no solo tiene consonancia con la estructuración de la sexualidad en Occidente de acuerdo a Foucault (2009), sino que además es parte de las exigencias actuales de grupos conservadores cuando se posicionan en contra de la ideología de género (Sequeira, 2019). 
Durante los años setenta y ochenta, tanto el Centro de Orientación Familiar (COF), adscrito a la iglesia anglicana, como el Centro de Integración Familiar (CIF) y el Movimiento Familiar Cristiano (adscritos a la iglesia católica) tuvieron interés en desarrollar capacitaciones sobre reproducción y sexualidad. De hecho, en las décadas finales del siglo $X X$, inclusive el OPUS DEI de Costa Rica, empleó el método de hacer programas radiales para instruir a sus feligreses en temáticas relativas a la sexualidad, siguiendo una exitosa fórmula que había empleado el COF (Cortés et al., 2008).

Más adelante, en las décadas de 1980 y 1990, las preocupaciones relacionadas a evitar la propagación de la epidemia del SIDA, así como la necesidad de disminuir los embarazos en adolescentes y niñas, también propiciaron una exigencia de diversos sectores por una mayor y mejor educación sexual en las escuelas y colegios públicos del país. En todo caso, para estas tres décadas finales, se pueden apreciar tiranteces constantes entre sectores religiosos y gubernamentales para influir en contenidos y demás aspectos de la educación sexual. En dichas disputas, a veces incomodaban los términos empleados, las referencias a ciertas instancias, la cantidad o profundidad de algunos vocablos que aludían a un cierto aspecto (los anticonceptivos, por ejemplo), la falta de referencia a situaciones o emociones (matrimonio, Dios, castidad o amor) (Faerrón, 2002) o inclusive los dibujos, que parecían excesivos e inmorales (Martínez, 1992, p. 5A). Muchas modificaciones se hicieron con un malestar explícito o con una complaciente aprobación de parte de las autoridades del MEP o de quienes habían elaborado estos documentos (Gamboa, 2009; Faerrón, 2002).

\section{TENSIONES Y DEMANDAS EN EL NUEVO SIGLO}

A inicios del año 2000, algunas instituciones demográficas nacionales mostraban al país un panorama preocupante en el campo de la salud sexual y reproductiva, especialmente en la población adolescente y joven, caracterizado por altos índices de embarazo en adolescentes, un inicio más temprano de las relaciones sexuales, baja utilización de anticonceptivos en la primera relación sexual y otros comportamientos de riesgo (INEC, 2020; Chen et al., 2001). Todo parecía indicar que los esfuerzos por educar en sexualidad, iniciados en 1968 por el MEP, no estaban dando los resultados esperados (Defensoría de los Habitantes de la República, 2012). En ese momento, Costa Rica contaba con un marco jurídico amplio

98

Doris Fernández Carvajal, María Luisa Preinfalk Fernández, Paula Sequeira Rovira

\section{()(1)(2)}


para la protección de la niñez y adolescencia (Valverde, 2007) y a nivel internacional se había ratificado una serie de instrumentos que referían a los derechos sexuales y reproductivos, en general, y al derecho a la educación sexual en particular (Morales, 2013).

Las dos primeras décadas de este nuevo siglo serían una época de intensos debates nacionales acerca de temas como la educación para la sexualidad, el aborto, la fertilización in vitro, el matrimonio igualitario, entre otros. Diferentes actores sociales, como la Iglesia Católica, la Iglesia Evangélica y colectivos como Despierta Costa Rica y No a la ideología de género-Unidos por la familia, que llamaban al rescate de la familia tradicional (Cerdas, 2017), establecerían nuevas alianzas y tejerían estrategias para tratar de marcar el rumbo de la historia y detener las transformaciones sociales que se vislumbraban en materia de derechos sexuales y reproductivos. Resurgirían con mayor fuerza, tanto en el país como en América Latina, los denominados grupos pro-vida que, con el apoyo de organizaciones internacionales e instancias de poder político y económico, retomarían la noción de "ideología de género" y el supuesto peligro que representa para la familia tradicional y la vida, criticando el feminismo y el Enfoque de Género y conformando un movimiento que llegaría a socavar algunas iniciativas estatales (Alvarenga 2018; Sequeira, 2020a, 2020b). El Estado enfrentaría no solo las presiones de estos grupos, sino también las demandas de diversos colectivos que, desde el siglo anterior, venían clamando por el cumplimiento de la normativa nacional y las obligaciones emanadas de convenios internacionales, apelando a reiteradas llamadas de atención realizadas por organismos internacionales e instancias de control interno. A continuación, se retoman algunos aspectos de importantes acontecimientos en materias de la sexualidad y la reproducción de principios del siglo XXI (Defensoría de los Habitantes de la República, 2012; Mesa de Diálogo Sociedad Civil, 2016; Coalición feminista para el avance de los derechos de las mujeres, 2017).

En materia de educación sexual, los avances y retrocesos dejaban entrever posiciones irreconciliables, que reflejan el carácter estratégico de la educación sexual como instrumento de reconformación del tejido social (Laboratorio de Innovaciones Sociales, Educativas y Culturales, LISEC, 2007). El hecho de que Costa Rica sea un Estado confesional le impregna un carácter particular a esta historia, como se verá más adelante. 
En el año 2001, el Estado costarricense dio un paso trascendental en el campo de la educación sexual, al conferirle carácter de política pública, con la aprobación de las Políticas de Educación Integral de la Sexualidad Humana (Consejo Superior de Educación, 2001). Este instrumento se consideró revolucionario, ya que estableció la educación sexual obligatoria y transversal en el currículo de la Educación General Básica. Se pretendía, así, cumplir con la normativa nacional y compromisos internacionales en materia de derechos de la niñez y adolescencia, superar el enfoque biologicista de la sexualidad, que venía imperando -fragmentado y centrado en aspectos reproductivos-y trascender los alcances cortoplacistas de los proyectos o programas ministeriales de décadas anteriores, para dar paso a esfuerzos permanentes y de carácter intersectorial. Sin embargo, las expectativas que generaron estas políticas se desvanecerían para quienes esperaban un cambio de rumbo; aunque se promulgaron con un carácter secular, estaban matizadas por preceptos religiosos, lo cual no permitía superar el enfoque prevaleciente (Preinfalk-Fernádez, 2016).

Aunque el Estado hizo esfuerzos por operacionalizar estas políticas con la creación del Departamento de Educación Integral de la Sexualidad Humana, como instancia coordinadora y ejecutora de estas (MEP, 2001), al finalizar la primera década del siglo XXI las autoridades educativas recibieron un llamado de atención por parte de la Defensoría de los Habitantes, por el retraso de casi una década en su aplicación, que evidenciaba no solo el impacto de un debate político-religioso mal manejado, sino la imposibilidad de formar al personal docente en los nuevos enfoques (Defensoría de los Habitantes, 2012).

Iniciando la segunda década del nuevo siglo y en un contexto de presiones internas por parte de la sociedad civil en torno a este tema, el MEP presentó al país el Programa de Estudio de Educación para la Afectividad y la Sexualidad Integral (MEP, 2012), acción calificada por algunos sectores de esfuerzo histórico, por tratarse de una propuesta educativa laica, basada en un abordaje integral de la sexualidad y con los enfoques de derechos humanos y género como ejes transversales; además, respondía a las exigencias internacionales y a la legislación interna. La naturaleza del nuevo programa dejaba entrever una ruptura evidente entre el gobierno y la Iglesia Católica, que no compartía estos enfoques, pero esta vez, nuevos actores sociales se unieron a esta última, articulándose rápidamente un movimiento social que logró socavar la iniciativa estatal. La Sala 
Constitucional de la Corte Suprema de Justicia declaró "parcialmente con lugar" los recursos de amparo contra el MEP que interpusieron familias católicas y evangélicas y declaró el programa opcional (Preinfalk-Fernández, 2016). El resultado fue un programa educativo que aborda la sexualidad desde un enfoque integral, considerando las dimensiones afectiva, corporal y espiritual, pero que no llegó a todo el estudiantado, con el agravante de que la población docente no dominaba los contenidos propuestos, carecía de las destrezas necesarias para impartir las temáticas desde los nuevos enfoques y era necesario un cambio de mentalidad para eliminar mitos, prejuicios y estereotipos (León et al., 2013).

En el 2017, el MEP le dio un nuevo empuje al Programa, aprobando una propuesta que incorporaba, además, los enfoques de diversidad y educación inclusiva; sin embargo, las medidas de presión fueron múltiples y lograron debilitarla (Cerdas, 2017). Tras esta situación, una parte de la ciudadanía quedaba nuevamente con un disgusto en materia de educación sexual y frente a lo que consideran una deuda que el Estado no ha podido saldar en más de 50 años.

Otro de los debates que inició en las últimas décadas del siglo $X X$, y que continúa hoy en día, es el referido al aborto; un tema polémico, que cobra relevancia no solo por el peligro que representa para la vida de las mujeres si se practica en condiciones de riesgo, sino también por su magnitud y las implicaciones políticas que tiene. Datos de la Organización Mundial de la Salud indican que entre el 2010 y el 2014 se produjeron en el mundo 55.7 millones de abortos anuales, de los cuales el $45 \%$ se efectuó en condiciones de riesgo y un $97 \%$ de esos abortos peligrosos se realizaron en países en vías de desarrollo (Ganatra et al., 2017).

En Costa Rica, el aborto es catalogado como un delito contra la vida y se regula en el Código Penal. A partir de 1970 se tipifican cuatro clases de aborto y diversas penas que varían en función de factores como la edad de la mujer, el tiempo de gestación, si existe consentimiento o no por parte de la mujer, si está en riesgo su vida o si conduce a su muerte. Los tipos de aborto incluidos en el Código Penal son: aborto con o sin consentimiento (Artículo 118); aborto procurado (Artículo 119); aborto honoris causa (Artículo 120); aborto impune (Artículo 121); aborto culposo (Artículo 122). El único caso en que el aborto no es punible es cuando se efectúa con el fin de proteger la vida o la salud de la mujer ante posibles complicaciones 
con su embarazo, que se conoce como aborto terapéutico y se define en el Código Penal como aquel

practicado con consentimiento de la mujer por un médico o por una obstétrica autorizada, cuando no hubiere sido posible la intervención del primero, si se ha hecho con el fin de evitar un peligro para la vida o la salud de la madre y éste no ha podido ser evitado por otros medios (Artículo 121).

También, existen normas de excepción de la pena que refieren al Perdón judicial (Artículo 93 del Código Penal). Sin embargo, en la práctica las mujeres han enfrentado, hasta la fecha, limitaciones para acceder a un aborto impune o terapéutico en los servicios de salud, por la falta de una norma técnica y protocolos de atención que regulen y orienten los procedimientos, ya que la aplicación de la norma depende de la interpretación del personal médico y obstétrico que, en la mayoría de los casos, al parecer por prejuicios, creencias personales o temor a ser demandado judicialmente por su actuación, se niega a practicarlo cuando está en riesgo la vida de la mujer, como lo evidencian las demandas interpuestas en 2007 y 2012 al Estado costarricense, ante la Comisión Interamericana de Derechos Humanos (CIDH), por dos mujeres a quienes se les negó este derecho (Center for Reproductive Rights, 2019).

Se espera que cambie esta situación con la aprobación del Decreto Ejecutivo Número 42113-S12, firmado en diciembre de 2019 por el Presidente de la República y el Ministro de Salud, en el cual oficializan la "Norma técnica para el procedimiento médico vinculado con el Artículo 121 del Código Penal" (La Gaceta, 17 de diciembre de 2019), así como con la aprobación, por parte del Ministerio de Salud, en diciembre 2020, del Protocolo de atención clínica para el procedimiento médico vinculado con el artículo 121 del Código Penal: interrupción terapéutica del embarazo (Ministerio de Salud, 2020).

Al tratarse de una práctica clandestina, es difícil estimar el número de abortos que se practican en el país. Cifras de la CCSS indican que, durante el período 1997 al 2017, se registraron 80 intervenciones en 16 hospitales estatales, lo que equivale a un promedio de 4 abortos terapéuticos por año (Ruiz, 2018). Sin embargo, estimaciones de la Asociación Demográfica Costarricense (ADC, 2008) muestran que, a inicios de la primera década 
del siglo XXI, en el país se efectuaban aproximadamente 27000 abortos inducidos anualmente. Estos datos, que posiblemente estén subestimados por el carácter punitivo que recae sobre esta práctica, muestran el poco control sobre la reproducción que tienen las mujeres en Costa Rica, pese a que la libertad y autonomía reproductiva constituye un derecho ampliamente protegido por la legislación nacional e internacional. Cifras de la Encuesta Nacional de Salud Sexual y Reproductiva 2010 indican que un $47.0 \%$ de las mujeres en edad fértil no deseaba su último embarazo, mientras que en los hombres el porcentaje fue del 38.3\%; tendencia que se mantuvo en los años previos a la encuesta (Ministerio de Salud, 2011).

Costa Rica ha ratificado diversos instrumentos internacionales que abordan de forma directa o indirecta el tema de los derechos sexuales y reproductivos, pero no había sido capaz de cumplir con sus obligaciones como Estado Parte en lo que al aborto terapéutico se refiere (ONU, 1999; ONU, 2016). Este incumplimiento llevó a que el Estado costarricense fuera demandado ante la $\mathrm{CIDH}$, como se indicó anteriormente, por Ana y Aurora, quienes por motivo de seguridad utilizan pseudónimos y alegaron afectación de sus derechos, al negárseles la interrupción de su embarazo en el sistema de salud del país, aun cuando el feto presentaba malformaciones no compatibles con la vida extrauterina y existía un riesgo para su salud (Center for Reproductive Rights, 2019). En respuesta a estas denuncias, en el 2015 el Estado costarricense manifestó su anuencia a desarrollar un "procedimiento de solución amistosa", aceptando que había violentado los derechos de las demandantes y comprometiéndose a elaborar las regulaciones necesarias que dieran contenido a la figura del aborto terapéutico en el sistema de salud. Sin embargo, en el 2018, a escasos días de concluir la administración del presidente Solís Rivera, se emitió un comunicado indicando que por "decisión política" se suspendía de manera indefinida la aprobación de la norma, ya que no existían "las condiciones políticas" para concretarla. Posteriormente, el presidente Carlos Alvarado, en campaña electoral, reconoció la necesidad de contar con lineamientos y protocolos para aplicar el Artículo 121 del Código Penal (Murillo, 2018), pero en negociaciones ante una segunda ronda electoral, se comprometió a no realizar modificaciones (Valverde, 2018).

Este hecho fue el detonante para la conformación de un movimiento denominado Aborto Legal Costa Rica (2018), que agrupó a más de 50 colectivos y activistas independientes, con el objetivo común de que se 
despenalizara y legalizara el aborto seguro y gratuito a todas las mujeres en el país considerando su derecho a decidir libremente sobre su cuerpo. Estas iniciativas se insertan en un movimiento que se ha venido articulando, a nivel de América Latina, a favor del derecho al aborto, impulsado por grupos feministas; desde la década de los años ochenta, a partir de procesos de democratización y, en los años noventa, en el marco de reformas constitucionales y judiciales, sucedidos en varios países de la región (Rubial, 2018). Otra parte de la población costarricense, que se manifestó en contra del aborto, se aglutinó en torno a los denominados "grupos pro-vida" ${ }^{1}$, y articula intereses religiosos (iglesia católica y representantes de otras denominaciones religiosas) con intereses políticos (diputados de orientación cristiana y evangélica). Este grupo abogó por que no prosperara la definición de la norma técnica, la normativa nacional fuera más restringida en situaciones de aborto y se desarrollaran políticas a favor de la familia (Vega, 2018).

Pese a estas presiones, el Presidente Alvarado firmó la norma técnica y se aprobó el protocolo de atención clínica, acciones que denotan un avance del país en el cumplimiento de los Derechos Sexuales y Reproductivos, pero que también generan nuevos retos para su cumplimiento efectivo en un contexto de polarización.

\section{CONCLUSIONES}

Tal y como se ha señalado en este artículo, la contextualización de la sexualidad es fundamental para entender el pasado, presente y futuro de las personas como sujetos sexuales. Desde el siglo XIX, los países europeos comenzaron a producir nociones y debates sobre la sexualidad que, posteriormente, serían exportados a otros países. Se producen intereses sobre la administración de la sexualidad en las mujeres, en los menores de edad, en las parejas y aquellos a quienes se suponía practicaban conductas inadecuadas (Foucault, 2009). Con el pasar del tiempo, este interés por la sexualidad empezará a provocar el paso de una concepción esencialista basada en un determinismo biológico, a una visión construccionista que, si bien considera el aspecto biológico como un componente importante, agrega otras dimensiones a su análisis, como serían la económica, política

1 El movimiento pro-vida existe a nivel mundial y data de los años 70 . Su origen se ubica en Estados Unidos a partir de la sentencia Roe v. Wade, de 1973, en la que la Corte Suprema de los Estados Unidos reconoció el derecho al aborto inducido.

104

Doris Fernández Carvajal, María Luisa Preinfalk Fernández, Paula Sequeira Rovira

\section{()(1)(2)}


e histórica. Lo anterior le provee al estudio de la sexualidad un contexto social-cultural que le brinda mayores elementos para una mejor comprensión, de su pasado, su presente y su futuro.

En este contexto, deben analizarse los ejes sobre los cuales han girado diversos debates en materia de sexualidad y que tuvieron lugar en el país durante el período analizado. Uno de esos ejes es el relativo a la reproducción humana; este ha tenido un lugar protagónico en la visión de desarrollo de Costa Rica, como se ha mostrado en los planteamientos anteriores. La institucionalización de la sexualidad, a finales de la década de 1960, trajo consigo un mayor interés por la educación sexual, asociada a la intención por parte de las naciones desarrolladas del mundo occidental de impulsar cambios en los patrones reproductivos en aquellos países considerados subdesarrollados. Dentro de esta perspectiva, el crecimiento poblacional desmedido era visto como un obstáculo en la superación del subdesarrollo económico.

Estas tesis de control natal, enmarcada en posiciones neo maltusianas (Malthus, 2007), consideraba que el crecimiento poblacional se extendía a un ritmo mayor que la producción de alimentos, lo que traería como consecuencia, entre otras, escasez de suministros, mal nutrición y enfermedades, situaciones poco deseables en la superación del subdesarrollo. Prueba de ello es que esta propuesta impulsada en países subdesarrollados contó con el financiamiento de naciones desarrolladas y otras organizaciones privadas como las ya citadas, que destinaron para ese fin importantes recursos económicos para su operacionalización.

En el período de estudio, aparecen en Costa Rica las categorías explosión demográfica y, posteriormente, la de planificación familiar, las cuales perdurarán aproximadamente por tres décadas. Estas ponían el acento en el cuidado al crecimiento poblacional desmedido, recomendando para ello el uso de anticoncepción femenina. El término de "planificación familiar", aun hoy día, es utilizado por los sectores religiosos más conservadores del país.

Es a mediados de la década de los noventa cuando surgen las categorías de "derechos reproductivos" y "derechos sexuales" en un marco social y político diferente, relativo al de derechos humanos, donde se busca que las mujeres asuman las decisiones sobre la reproducción con mayor 
información y autonomía sobre su cuerpo. En este sentido, la Conferencia Internacional sobre Población y Desarrollo, realizada en El Cairo en el año 1994, ofrece el marco para mejorar la situación de mujeres, en especial de niñas y adolescentes, en materia de salud sexual y reproductiva. Esto es importante porque, por un lado, deja atrás la propuesta del control natal como medio de superación del subdesarrollo para empoderar a las mujeres niñas y adolescentes como sujetas de derechos en materia de salud sexual y reproductiva; $y$, por otro, constituye una estrategia en la reducción de la pobreza y el mejoramiento de la salud y calidad de vida.

Es importante resaltar que la propuesta de educación en sexualidad, como medio de control natal en Costa Rica, ha estado marcada por tensiones entre instituciones laicas que la promueven a través de diferentes medios, programas radiales y capacitaciones y las posiciones religiosas provenientes de la iglesia católica y organizaciones evangélicas, quienes han visto en esta iniciativa un problema derivado de la práctica de una sexualidad sin control o la incitación a la vivencia de la sexualidad con "libertinaje", sobre todo por parte de las mujeres.

Asimismo, la institucionalización de la educación en sexualidad que se experimenta en el período de análisis se expresa en dos planos. En primer lugar, cuando el Estado costarricense asume funciones y posiciones en torno al tema y, en segundo lugar, el surgimiento de organizaciones civiles. En la primera situación aparecen los primeros programas por parte del Estado que se instalan en el MEP. Estos tienen como finalidad el impulsar acciones para educar a la niñez y adolescencia en materia de sexualidad. Por otro lado, se tiene el surgimiento de diversas organizaciones no gubernamentales, que logran crearse y existir por varios años, gracias al respaldo económico del exterior, con la intencionalidad antes descrita.

Tanto por parte del Estado como por las organizaciones civiles, se logra colocar una agenda en materia de sexualidad, tendiente al control sexual y reproductivo de las mujeres, primeramente, sustentado en el control natal con miras a un mejor desarrollo socioeconómico y, posteriormente, justificado en la disminución del embarazo a temprana edad en niñas y adolescentes, por las consecuencias sociales y económicas que esto conlleva. 
Además de los anteriores, el tema del aborto en Costa Rica es otro eje de discusión que se posiciona en el período de estudio. Este también ha estado cargado de gran tensión entre grupos y organizaciones civiles, que lo ven como un derecho humano, y grupos religiosos católicos y evangélicos pentecostales considerados pro-vida, con una posición radicalmente opuesta. Se mencionó que el aborto es reconocido como un delito según el Código Penal, excepto si se practica cuando se encuentra en riesgo la salud o la vida de la mujer; no obstante, ocurren en el país un número indeterminado de abortos inducidos, considerados clandestinos, poniendo en riesgo la salud y la vida de quienes se someten a estos procedimientos.

Tanto la educación en sexualidad como la anticoncepción y el aborto continúan siendo temas vigentes en Costa Rica; siguen siendo sometidos a grandes tensiones, pues unos grupos pugnan por su reconocimiento como derechos humanos, mientras que otros pujan por contrarrestar la influencia de las concepciones adyacentes a los derechos sexuales y derechos reproductivos. Todo esto hace que la sexualidad y la reproducción de las mujeres continúen siendo temas de gran contenido político. Dado lo anterior, se hace necesario dar seguimiento a las discusiones que puedan darse en el corto y en el mediano plazo, para determinar por dónde se decantan y las consecuencias que podrían tener para el bienestar de las personas.

\section{BIBLIOGRAFÍA}

Aborto Legal Costa Rica. (2018). Movimiento por el aborto legal, seguro y gratuito en Costa Rica. https://www.abortolegalcr.com/

Allen, A. (2001). Pornography and Power. Journalof Social Philosophy, 32(4), 512-531. http://web.a.ebscohost.com.una.remotexs.co/ehost/pdfviewer/pdfviewer?vid=1\&sid=f17230f0-1cc0-4a7e-af63-24f8ca8a9ad8\%40sessionmgr4008

Alvarenga, P. (2018). Identidades y política en la era de los fundamentalismos. Praxis. Revista de Filosofía, (78), 1-10. https://doi.org/10.15359/ praxis. 78.1 
Asociación Demográfica Costarricense (ADC). (1987). Encuesta Nacional de Fecundidad y Salud Costa Rica 1986. Autor.

Asociación Demográfica Costarricense (ADC). (2008). Estimación del aborto inducido en Costa Rica, 2007. http://www.adc-cr.org/aborto_ inducido.pdf

Barth, L. (1988). Los jóvenes y la educación para la sexualidad: una alternativa metodológica. [Tesis de Licenciatura] Universidad Nacional de Costa Rica.

Carcedo, A., Sagot, M., \& Trejos, M. (1997). Improving the quality of women's daily lives. In: I. A. Leitinger (Ed.), The Costa Rican Women's Movement. A reader (pp. 19-23). University of Pittsburgh Press.

Carranza, M. (2004). Sobre una relación «prolífica». El papel de «la salud» en la propagación de la esterilización contraceptiva en Costa Rica. Dynamis: Acta Hispanica ad Medicinae Scientiarumque Historiam Illustrandam, 24, 187-212. https://www.raco.cat/index.php/Dynamis/ article/view/113912

Castro, J. (2015). Estados Unidos y la Guerra por el desarrollo: el control de la natalidad en Chile, 1960-1970. Revista Complutense de Historia de América, 41, 95-120. https://revistas.ucm.es/index.php/RCHA/ article/view/49898/46371

Center for Reproductive Rights. (2019). Derechos a la salud de las mujeres embarazadas. https://www.reproductiverights.org/sites/crr.civicactions.net/files/documents/Aurora\%20Fact\%20Sheet\%20Final.pdf

Cerdas, D. (2017, 16 de julio). MEP invertirá $\phi 800$ millones anuales en psicólogos para impartir nueva asignatura de Sexualidad. La Nación. https://www.nacion.com/el-pais/educacion/mep-invertira-c-800-millones-anuales-en-psicologos-para-impartir-nueva-asignatura-de-sexualidad/5M62DYE5FVCPNK6U4CJS3RQNWY/ story/ 
Chen, M., Rosero, L., Brenes, G., León, M., González, M. I., \& Vanegas, J. C. (2001). Salud reproductiva y migración nicaragüense en Costa Rica 1999-2000: resultados de una Encuesta Nacional de Salud Reproductiva. Programa Centroamericano de Población e Instituto de Investigaciones en Salud, Universidad de Costa Rica. http://www. kerwa.ucr.ac.cr/handle/10669/75351

Coalición feminista para el avance de los derechos de las mujeres. (2017). Informe alternativo sobre el cumplimiento del Estado de Costa Rica para el Comité de las Naciones Unidas para la Eliminación de la Discriminación contra la Mujer (CEDAW/C/67/1). https://clacaidigital.info/bitstream/handle/123456789/1001/INT_CEDAW_NGO_ CRI_27705_S.pdf?sequence=5\&isAllowed $=y$

Connelly, M. (2008). Fatal misconception. The struggle to control world population. Belknap Press.

Consejo Superior de Educación (2001). Política de Educación Integral de la Sexualidad Humana. Republica de Costa Rica

Cortés, A., Bernal, R., Urrutia, J., Alvarenga, M. J., Trejos, R., \& Ramos, J. A. (2008). La Iglesia Anglicana en Costa Rica: perspectivas históricas. [Trabajo final de graduación de licenciatura]. Universidad Nacional de Costa Rica.

Defensoría de los Habitantes de la República. (2012). Educación para la sexualidad humana: un derecho fundamental de la niñez y la adolescencia que el Ministerio de Educación Pública debe garantizar. En: V. Muñoz y C. Ulate (Eds.), El derecho humano a la educación para la afectividad y la sexualidad integral: contribuciones para una reforma educativa necesaria (pp. 100-124). Universidad Nacional de Costa Rica, Doctorado en Estudios Latinoamericanos, Instituto de Estudios de la Mujer. https://www.adc-cr.org/pdf/educacion_sexual_digital.pdf

Dirección General de Estadística y Censos. (1978). Encuesta Mundial de Fecundidad de la República de Costa Rica, 1976. Dirección General de Estadística y Censos. 
Ehrlich, P. R. (1969). The Population Bomb. (Fifth Printing). Ballantine Book.

Elizondo A, J., Shultz, J. M., Baum, M. K., \& Herrera M. G. (1993). Epidemiología del SIDA y de la infección por el VIH en Costa Rica. Bulletin of the Pan American Health Organization, 115(3), 195-214. https://iris.paho.org/bitstream/handle/10665.2/16314/v115n3p195. pdf? sequence=1\&isAllowed=y

Faerrón, A. L. (2002). La Educación para la Sexualidad en el contexto de la sociedad costarricense: análisis del proceso de diseño y gestión del Programa Amor Joven (1998-2000). [Tesis de Maestría]. Universidad de Costa Rica-Universidad Nacional de Costa Rica.

Felitti, K. (2009). Derechos reproductivos y políticas demográficas en América Latina. Íconos. Revista de Ciencias Sociales 13(3), 55-66. http://200.41.82.22/bitstream/10469/915/4/RFLACSO-I35-05-Felitti. pdf

Fernández, M. (1992, 25 de enero). El viacrucis de las guías sexuales. La República.

Fernández, D., \& Sequeira, P. (2011). Diez años después de aprobada la ley del derecho a la esterilización en Costa Rica. Revista Costarricense de Salud Pública, 2(20), 107-118.

Foucault, M. (2001). Los anormales. Curso del Collège de France (19741975). Ediciones Akal.

Foucault, M. (2009). Historia de la Sexualidad I. La Voluntad de Saber. Siglo XXI.

Gamboa, I. (2009). En el Hospital Psiquiátrico. El sexo como locura. Grafos Litografía.

Ganatra, B., Gerdts, C., Rossier, C., Johnson, B. R., Tunçalp, Ö., Assifi, A., Alkemaet, L. (2017). Global, regional, and subregional classification of abortions by safety, 2010-14: estimates from a Bayesian hierarchical model. The Lancet 390(10110), 2333-2334. https://doi. org/10.1016/S0140-6736(17)31794-4 
González, L. (1991, 2 de diciembre). Las guías de educación sexual ¿para quiénes son? La República.

González, M. L. (1985). Planificación Familiar y el Estado. El caso de Costa Rica. [Tesis de Licenciatura]. Universidad de Costa Rica.

Instituto Nacional de Estadística y Censos (INEC). (2020). Nacimientos. https://www.inec.cr/poblacion/nacimientos

La Gaceta. (2019, 17 de diciembre). Decreto Ejecutivo Número 42113-S. Alcance 281 a La Gaceta 240. Imprenta Nacional de Costa Rica.

La República. (1970, 20 de marzo). Educación sexual y Demografía enseñarán en Costa Rica. La República.

La República. (1970, 10 de abril). Curso de demografía y educación sexual a nivel centroamericano. La República.

La República. (1977, 11 de setiembre). Esto es el CIF. La República.

Laboratorio de Innovaciones Sociales, Educativas y Culturales (LISEC). (2007). Indagaciones en torno a la problemática de la sexualidad en el terreno de la educación. Programa Nacional de Salud Sexual y Procreación Responsable. República de Argentina. http://www.lisec. org.ar

León, G., Bolaños, G., Campos, J., \& Mejías, F. (2013). Percepción de una muestra de educandos y docentes sobre la implementación del programa educación para la afectividad y la sexualidad integral. Revista Electrónica Educare, 17(2), 145-165. http://www.revistas.una.ac.cr/ index.php/EDUCARE/issue/current

Madrigal, J., \& Sosa, D. (1987). Antecedentes del estudio. Encuesta Nacional de Fecundidad y Salud Costa Rica 1986. Asociación Demográfica Costarricense.

Malthus, T. (2007). Ensayo sobre el principio de la población. Claridad. 
Maroto, L., Morales, R., \& Sequeira, P. (2004). Influencia de la cultura, la religión y la institución médica en la decisión de las mujeres a la hora de realizarse la esterilización femenina: Un estudio de caso en el Hospital San Juan de Dios. [Tesis de Licenciatura]. Universidad de Costa Rica.

Martínez, L. (1992, 12 de julio). Suprimen dibujos de guías sexuales. La República.

Mesa de Diálogo de laSociedad Civil. (2016). Informealternativo:observaciones de las Organizaciones de la Sociedad Civil frente al sexto informe periódico que los Estados partes debian presentar en el 2012. https:// clacaidigital.info/bitstream/handle/123456789/1196/examen\%20estados\%20parte\%20articulo\%2040. pdf?sequence=2\&isAllowed=y

Ministerio de Educación Pública (MEP) (2001). Políticas de Educación Integral de la Sexualidad Humana. Sesión No. 28-01 del día 12 de junio de 2001. Autor.

Ministerio de Educación Pública (MEP). (2012). Programa de Estudio de Educación para la Afectividad y la Sexualidad Integral. Autor.

Ministerio de Salud. (2011). Informe de los resultados de la Encuesta de Salud Sexual y Reproductiva 2010. Ministerio de Salud.

Ministerio de Salud. (2016). Il Encuesta Nacional de Salud Sexual y SaIud Reproductiva 2015. Ministerio de Salud/Asociación Demográfica Costarricense/Centro Centroamericano de Población/Fondo de Población de las Naciones Unidas (UNFPA).

Ministerio de Salud. (2020). Ministerio de Salud aprueba protocolo de atención de la CCSS para interrupción terapéutica del embarazo. https://www.ministeriodesalud.go.cr/index.php/centro-de-prensa/noticias/741-noticias-2020/2002-ministerio-de-salud-aprueba-protocolo-de-atencion-de-la-ccss-para-interrupcion-terapeutica-del-embarazo

Morales, M. F. (2013). Los derechos sexuales y reproductivos: Estudio y análisis legal del aborto no punible en Costa Rica. [Tesis de Licenciatura]. Universidad de Costa Rica. 
Murillo, A. (2018, 31 de julio). Calientan de nuevo: Aborto terapéutico y derechos LGTBI en momento clave. Semanario Universidad. https:// semanariouniversidad.com/pais/calientan-de-nuevo-aborto-terapeutico-y-derechos-Igtbi-en-momento-clave/

Organización de las Naciones Unidas (ONU). (1999). Examen de los informes presentados por los Estados partes en virtud del artículo 40 del Pacto Internacional de Derechos Civiles y Políticos. Comité de Derechos Humanos. https://tbinternet.ohchr.org/_layouts/15/TreatyBodyExternal/countries.aspx? Lang=sp

Organización de las Naciones Unidas (ONU). (2016). Examen de los informes presentados por los Estados partes en virtud del artículo 40 del Pacto Internacional de Derechos Civiles y Políticos. Comité de Derechos Humanos. https://tbinternet.ohchr.org/_layouts/15/TreatyBodyExternal/countries.aspx? Lang =sp

Preinfalk-Fernández, M. L. (2016). La Educación Sexual en el Sistema Educativo Formal Costarricense. Revista Tempos e Espaços em Educação, 9(19), 103-112. https://doi.org/10.20952/revtee.v9i19.5599

Rodríguez de Ortega, V. (1978). Antecedentes del estudio. Encuesta Mundial de Fecundidad de la República de Costa Rica, 1976. Dirección General de Estadística y Censos.

Rosero, L. (1981a). Dinámica demográfica, planificación familiar y política de la población en Costa Rica. Demografía y economía, 15(1), 59-84. https://estudiosdemograficosyurbanos.colmex.mx/index.php/ edu/article/view/519/512

Rosero, L. (1981b). Fecundidad y anticoncepción en Costa Rica 1981: Resultados de la Segunda Encuesta de Prevalencia Anticonceptiva. Asociación Demográfica Costarricense.

Rubial, A. (20 de marzo, 2018). Batalla legal feminista por el derecho al aborto en América Latina. OpenDemocracy.net. https://www.opendemocracy.net/es/movilizaci-n-legal-feminista-por-el-derecho-al-aborto-en-am-rica-latina/ 
Ruiz, P. (2018, 31 de agosto). Buscan prohibir vasectomías y venta de anticonceptivos. CRHoy. https://www.crhoy.com/nacionales/ buscan-prohibir-vasectomias-y-venta-de-anticonceptivos/

Sandoval, I., \& López, N. (1990). Embarazo adolescente en el Área Metropolitana de San José. Universidad Nacional de Costa Rica, Instituto de Estudios en Población.

Sequeira, P. (2019). Sexualidad, educación y locura. El conocimiento homosexualizador y la ideología de género en los programas de educación sexual del MEP. En: Ideología de género en Costa Rica. Tres ensayos (pp. 41-185). SEBILA.

Sequeira, P. (2020a). La sexualidad como suceso. Análisis de la percepción periodística de la homosexualidad entre mediados de 1965 y finales de 1980. Diálogos Revista Electrónica de Historia, 21(2), 66-84. https://www.scielo.sa.cr/pdf/dreh/v21n2/1409-469Xdreh-21-02-00066.pdf

Sequeira, P. (2020b). Los hippies como metáfora de la ambigüedad o del por qué se los responsabiliza por el surgimiento de la "ideología de género" en Costa Rica. Cuadernos Intercambio sobre Centroamérica y el Caribe, 17(2), e43520. https://doi.org/10.15517/c.a..v17i2.43520

Tone, A. (2002). Devices and desires: a history of contraceptives in America. Hill and Wang.

Valverde, O. (2007). Evaluación de Políticas Nacionales de Juventud en Costa Rica. Organización Iberoamericana de la Juventud.

Valverde, R. (2018, 30 de abril). Gobierno se aferra al silencio sobre razones para suspender norma sobre aborto terapéutico. Semanario Universidad. https://semanariouniversidad.com/ultima-hora/gobierno-se-aferra-al-silencio-sobre-razones-para-suspender-norma-sobre-aborto-terapeutico/

Valverde, L., De Rivera, A., \& Orozco, Á. (1991, 18 de diciembre). Iglesia rechaza guías de educación sexual. La República. 
Vega, A. (2018, 15 de mayo). El miedo y la mentira en la campaña electoral del 2018. Semanario Universidad. https://semanariouniversidad.com/ opinion/ el-miedo-y-la-mentira-en-la-campana-electoral-del-2018/

White, L., Gómez, V. M., \& Morris, L. (1992). Comportamiento sexual de los costarricenses menores de 25 años. Encuesta Nacional de Salud Reproductiva de Adultos Jóvenes. Caja Costarricense de Seguro Social. 
